\title{
EVALUACIÓN DE LA SUSTENTABILIDAD EN AGROECOSISTEMAS
}

\author{
ASSESSMENT OF THE SUSTAINABILITY OF AGROECOSYSTEMS \\ Toro, P. ${ }^{1 \star}$, García, A. ${ }^{2}$, Gómez-Castro, A.G. ${ }^{2}$, Perea, J. ${ }^{2}$, Acero, R. ${ }^{3}$ y Rodríguez-Estévez, V. ${ }^{2}$ \\ ${ }^{1}$ Pontificia Universidad Católica de Chile. Facultad de Agronomía e Ingeniería Forestal. Av. Vicuña Mackenna \\ 4860. Santiago. Chile. *pmtoro@uc.cl \\ ${ }^{2}$ Departamento de Producción Animal. Facultad de Veterinaria. Universidad de Córdoba. Campus de \\ Rabanales. 14014 Córdoba. España. \\ ${ }^{3}$ Departamento de Organización de Empresas. Universidad de Córdoba. Campus de Rabanales. 14014 \\ Córdoba. España.
}

\section{Palabras clave adicionales \\ Indicador. Índice. Metodología.}

\section{RESUMEN}

La sustentabilidad requiere ser valorada con el propósito final de implementar técnicas o tecnologías que mejoren o minimicen el impacto ambiental, para ello deben emplearse escalas de medición ordinales, que permitan su categorización. Indicadores e índices surgen como la base de las metodologías de evaluación de sustentabilidad, debiendo cumplir una serie de requisitos para ser de utilidad. Entre estos requisitos, la integración de las tres dimensiones de la sustentabilidad (económica, social y ambiental) es básica.

La evaluación se sustenta en el empleo de indicadores que describen una característica del estado de un sistema, generalmente a través de datos observados o estimados, e índices que consisten en una agregación cuantitativa de indicadores. Los indicadores se generan del análisis de los procesos que utilizan recursos ambientales para producir resultados y pueden ser medioambientales o de sustentabilidad (cuando se tienen en cuenta los factores de tiempo, límites y objetivos)

Índices como la Huella Ecológica, el Índice de Desarrollo Humano, el Producto Interno Bruto, entre otros informan tanto del grado de inequidad global como de la sobreutilización de recursos por parte de las economías de algunos países, Pero también con aplicación a sectores productivos, como por ejemplo a la ganadería.

\author{
ADDITIONAL KEYWORDS \\ Indicator. Index. Methodology.
}

En agroecosistemas se ha definido un gran número de indicadores dependientes del sistema en estudio, la mayor parte centrados en la productividad o los retornos económicos de la explotación. Tanto ecuaciones de regresión como modelos de simulación han sido utilizados para la estimación de indicadores, debido a la dificultad de obtención de medidas directas o con el propósito de descubrir qué efectos tendrá en el futuro una medida adoptada hoy.

\section{SUMMARY}

Sustainability needs to be assessed by means of ordinal scales, allowing their categorization. Indicators and indexes have emerged as the basis for sustainability assessment methodologies; they have had to meet certain requirements to be useful. Among these requirements, the integration of the three dimensions of sustainability (economic, social and environmental) is essential.

The evaluation is based on the use of indicators that describe a feature of the state of a system, generally through observed or estimated data, and indexes that consist of a quantitative aggregation of indicators. The indicators are generated from analysis of processes that uses environmental resources to produce results and they can be environmental or sustainability (when the factors of time, limits and objectives are considered). 
Indexes such as the Ecological Footprint, Human Development Index, Gross Domestic Product, among others give an idea on the extent of global inequality and the overuse of resources by the economies of some countries, but also with application to productive sectors, such as stockbreeding.

A large number of indicators in agroecosystems have been defined depending on the system under study, most focusing on economic returns or productivity of the farm. Both regression equations and simulation models have been used to estimate indicators, due to the difficulty of obtaining direct measures or for the purpose of discovering the futures effects of action taken today.

\section{INTRODUCCIÓN}

La sustentabilidad es un concepto complejo, controversial, multidimensional y en evolución, a partir del cual se evidencia la necesidad imprescindible de un proceso de transformación estructural, que permita integrar medioambiente con desarrollo, y a la vez economía con ecología. La necesidad de este proceso de transformación se deriva del mal uso de los recursos por el hombre, generado por el cambio social global debido al aumento de la población, el crecimiento económico, el avance tecnológico y la pobreza (Jiménez-Herrero, 1989; Bell, 1998).

La preocupante insostenibilidad del actual modelo de desarrollo humano ha generado la toma de conciencia junto con la necesidad de investigar sobre cómo evaluar y medir el estado de sostenibilidad (o insostenibilidad) de cada modelo de desarrollo y producción a distintas escalas, con el propósito final de implementar técnicas o tecnologías que mejoren o minimicen el impacto ambiental (FMAM, 2000). En otras palabras existe la necesidad de transformar el concepto de sustentabilidad en definiciones y estrategias operacionales que puedan ser utilizadas para evaluar el impacto de distintas acciones en la sustentabilidad de los sistemas (López-Ribaura et al., 2005).

La sustentabilidad está rápidamente pasando de un concepto abstracto a un estado medible de la dinámica de los sistemas humano-ecológicos (Mayer, 2008). Medidas generales de sustentabilidad como la Huella Ecológica, el Producto Interno Bruto, el PIB Verde, el Índice de Desarrollo Humano, entre otras, entregan información de la evolución de la sustentabilidad a distintas macro escalas, además de permitir la visualización de la inequidad entre naciones. Pero para abordar la evaluación de sustentabilidad en un agroecosistema es fundamental centrarse en las características básicas del concepto y determinar cómo son afectadas dentro de cada dimensión. De todas formas la medición de la sustentabilidad, según Masera et al. (1999) seguirá siendo una tarea compleja, debido a las siguientes razones:

- Diferentes escalas de evaluación que van desde el planeta hasta una granja.

- La multitud de estructuras productivas, cada una con sus interacciones, relaciones sinérgicas y de complementariedad que dificultan la estandarización.

- La necesidad de un punto de referencia que permita la comparación y emisión de juicios de valor sobre el grado de sustentabilidad de cada sistema, ya sea a través de la comparación transversal entre sistemas productivos o temporal de un sistema a lo largo de su evolución como consecuencia de la introducción de mejoras tecnológicas o prácticas de manejo.

- La necesidad de predecir el efecto en el grado de sustentabilidad futuro que tendrán las medidas adoptadas hoy.

En cualquier caso, la evaluación del grado de sustentabilidad se encuentra en la base de cualquier intento de mejora de los sistemas de producción. En agroecosistemas se han utilizado distintas metodologías para evaluar la sustentabilidad, desde unas muy detalladas, sólo aplicables a condiciones experimentales, a otras muy generales, pasando desde la simple toma de datos a campo, por encuestas y entrevistas y llegando hasta la predicción mediante ecuaciones de regresión y modelos de simulación. 
Se han realizado esfuerzos para implementar metodologías que sobrepasen algunas de las razones que dificultan la evaluación de sustentabilidad, por ejemplo, la metodología MESMIS (Marco de evaluación de sustentabilidad basado en indicadores), que identificando indicadores, dentro de cada uno de los siete atributos a través de los que define la sustentabilidad, permite realizar una evaluación adaptable a distintos sistemas de manejo de recursos naturales y sistemas agrícolas y ganaderos (Masera et al., 1999; López-Ridaura et al.,2002; López-Ridaura et al., 2005).

Sin embargo, tanto la complejidad como el alto grado de diversidad de los agroecosistemas y de las organizaciones socioeconómicas, hacen de la evaluación de la sustentabilidad una tarea complicada. En los últimos tiempos los distintos indicadores, índices y metodologías de evaluación han evolucionado y amplificado su potencia, mejorando la calidad de la información entregada y con ello la capacidad de conocer de modo más preciso lo que ocurre realmente con la sustentabilidad, tanto a escala local como global. En este trabajo se pretende considerar de modo sintético las herramientas disponibles para la evaluación de la sustentabilidad global, además de hacer una recopilación de aquellas más adecuadas para su aplicación en agroecosistemas pecuarios.

\section{EVALUACIÓN DE LA SUSTENTABILIDAD}

Según Kates et al., (2001), Devuyst et al., (2001) y Ness et al., (2007) el objetivo de la evaluación de sustentabilidad es proporcionar a los encargados de adoptar decisiones, una valoración de ámbito local a global que integre los sistemas de naturaleza y sociedad a corto y largo plazo, a fin de ayudar a determinar qué acciones deben o no deben ser emprendidas para favorecer una sostenible relación entre sistemas.

Cualquier metodología y sus indicado- res correspondientes precisan de modo indispensable una clara definición de los objetivos de la política en lo referente a la sustentabilidad (Singh et al., 2009).

En algunos casos, como cuando un recurso ofrece múltiples servicios (Popp et al., 2001), la medición de la sostenibilidad se hace aún más confusa, por lo que, para ellos, debe ser desarrollado un plan de gestión, que considere un índice de la calidad de los recursos para cada servicio.

Para realizar una evaluación de la sostenibilidad es necesario contar con información adecuada que se adapte a los objetivos cuantitativos de sustentabilidad y que debe, según Brink (1991):

a) proporcionar una indicación clara sobre el cumplimiento de los objetivos de la sustentabilidad

b) referirse al sistema en su conjunto

c) tener un carácter cuantitativo ficos

d) ser comprensible para los no cientí-

e) contener parámetros que puedan ser utilizados por períodos de una o más décadas.

De manera complementaria, Belloff y Beaver (2000), señalan que las medidas de sustentabilidad deberían satisfacer los siguientes criterios:

a) ser simples y comprensibles para una variedad de audiencias

b) ser reproducibles y consistentes al comparar diferentes períodos de tiempo, unidades de negocio, o alternativas de decisión

c) robustas y no contradictorias (es decir, una mejor medida debe indicar un rendimiento más sostenible)

d) complementar los programas de regulación existentes

e) ser costo-efectivas en términos de recogida de datos, haciendo uso en gran parte de los datos obtenidos o disponibles para otros fines

f) ser útiles para adoptar decisiones

g) ser apilables a lo largo de la cadena de suministro o del ciclo de vida del producto. 
Las metodologías de evaluación de la sustentabilidad se agrupan básicamente en dos tendencias apoyadas en las dos versiones de sustentabilidad (débil y fuerte). La primera de ellas, se caracteriza por una visión economista (sustentabilidad débil) y se basa en el método de agregación monetaria o contabilidad ambiental, mientras que la segunda (sustentabilidad fuerte), utilizada por científicos e investigadores de otras disciplinas, se basa en el uso de indicadores bio-físicos y, en algunos casos, su posterior agregación (Smith, 1996; Rapport et al., 1999).

El enfoque de contabilidad ambiental no implica que el simple registro contable del valor de los recursos y ecosistemas, haga visible una condición que permita establecer un juicio sobre la sustentabilidad. La contabilidad muestra los valores de las condiciones ambientales prevalecientes y los costos ocasionados con la alteración de los recursos en un período dado (López y Mantilla, 2006). Pero el problema es cómo debe valorarse monetariamente el medio ambiente natural, de modo que el mercado no se resienta y que al mismo tiempo pueda mejorarse o mantenerse el ecosistema. Este inconveniente de valoración medioambiental está íntimamente vinculado al principio de sustitución: para que el capital humano (inversión) pueda sustituir el capital natural hace falta monetarizar el medio ambiente (Luffiego y Rabadán, 2000).

Una dificultad adicional surge dado que, al igual que la aptitud en la evolución biológica, las valoraciones de la sustentabilidad sólo pueden hacerse a posteriori. Un organismo vivo es adecuado en la medida en que su progenie sobreviva y contribuya a la reserva de genes de las generaciones futuras. Para evaluar la aptitud de hoy en día se debe, por tanto, esperar hasta mañana. En el caso de la evaluación de la sustentabilidad, también se debe esperar hasta después de ocurridos los hechos (Costanza y Patten, 1995). En otras palabras, la sustentabilidad no es una situación de momento o una condición estática, sino que corresponde a un proceso verificable sólo con el transcurrir del tiempo, lo que implica que su evaluación es el resultado de análisis periódicos, en los cuales los registros contables se convierten en evidencias sustantivas, indicativas de dicha condición (López y Mantilla, 2006).

De este modo, la definición del grado de sustentabilidad es, a menudo, la predicción de que las medidas adoptadas hoy, conduzcan a la sustentabilidad en el futuro. Por ejemplo, el mantenimiento de tasas de recolección de un sistema de recursos por debajo de tasas de renovación natural, se podría suponer que conducen a un sistema de extracción sostenible, pero esto es una predicción, no una definición (Costanza y Patten, 1995), siendo además no del todo cierta, dada la existencia de ecosistemas, en los que la tasa de extracción debe ser equivalente a las de renovación para mantener el equilibrio. Así, por ejemplo, en la dehesa, una extracción inferior a la capacidad de sustentación conduce probablemente a una regresión hacia el bosque climácico mediterráneo, con lo cual, la capacidad productiva del sistema se degrada, hasta el punto de expulsar a la explotación ganadera. Otro ejemplo concreto se aprecia en la figura 1, que analiza el efecto del aumento del precio del agua en 1.0 Yuan $/ \mathrm{m}^{3}$ sobre algunos indicadores de sustentabilidad, como la sustentabilidad de las napas freáticas y la eficiencia de uso del agua, en un sistema de cultivo intensivo irrigado de trigo-maíz en la llanura norte de China, donde la gestión del agua y de los fertilizante nitrogenados son aspectos críticos para la gestión sostenible de recursos (Wei et al., 2009).

Phillis and Andriantiatsaholiniaina (2001), critican, las mediciones de sustentabilidad señalando, que "no sólo no existen unidades de medida comunes para los indicadores de la sustentabilidad, sino que además no se dispone de criterios cuantitativos para ciertos valores". De esta manera, se necesita, un método sistemático sobre la 


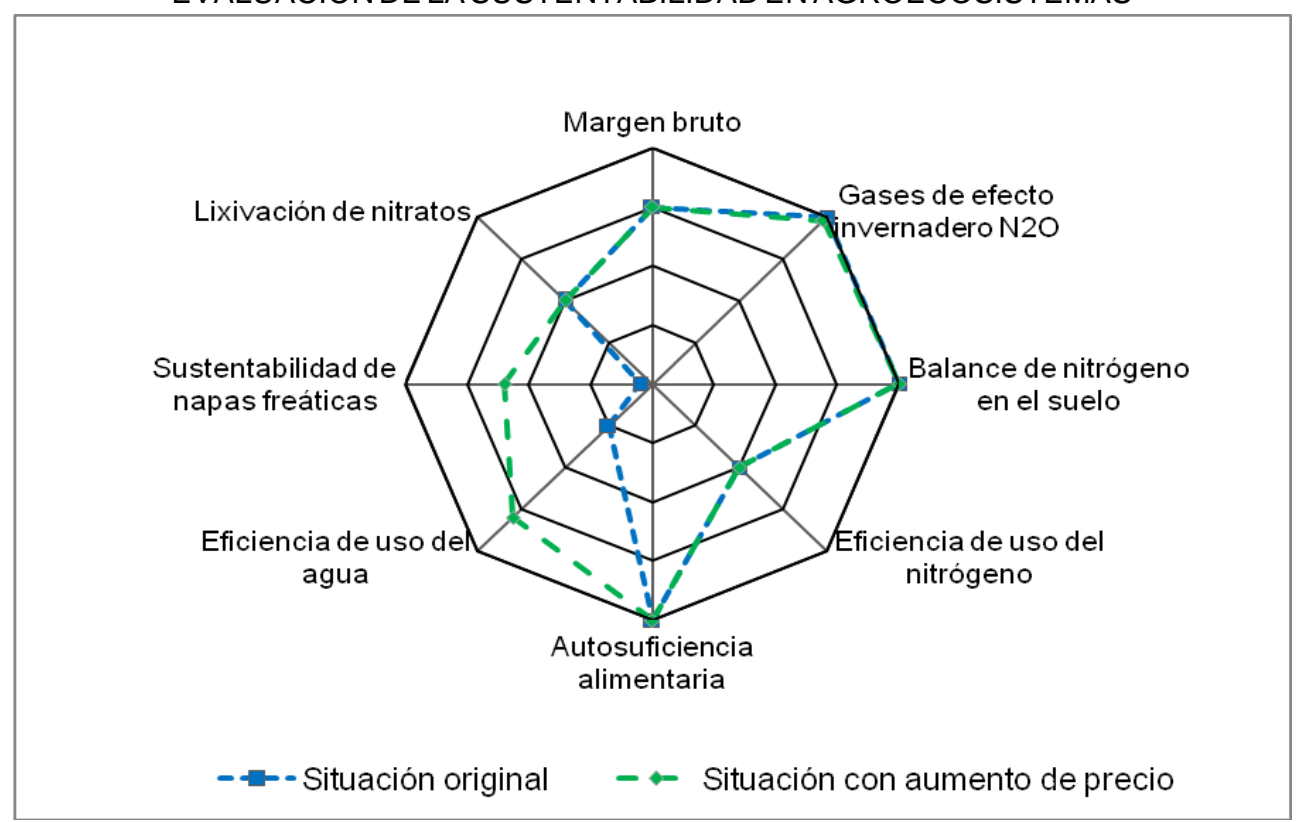

Fuente: Adaptado de Wei et al. (2009).

Figura 1. Efecto del cambio de precio del agua en indicadores de sustentabilidad. (Effect of change the price of water on indicators of sustainability).

base de una metodología científica confiable, que combine múltiples componentes y evalúe la incertidumbre y que, además, sea flexible y permita añadir y quitar indicadores para lograr una mejor evaluación del sistema de acuerdo al contexto. Rigby y Cáceres (2001), refiriéndose a las dificultades de la evaluación de la sustentabilidad en los sistemas agrícolas y ganaderos, señalan el inconveniente de las distintas unidades de medida y escalas de medición dependiendo de la dimensión de la sustentabilidad evaluada, a modo de ejemplo, mencionan la problemática de combinar en una medida global, los efectos de la producción orgánica en los márgenes de la explotación, la fertilidad del suelo y la generación de empleo rural. Además, una complicación adicional, surge si se consideran los trade-off, es decir, cuando un indicador de una dimensión aumenta y uno de otra cae.

En este ámbito, es propicio hacer mención a la ciencia post-normal, la que tiene relación con la necesidad de tomar decisiones cuando: los factores son inciertos, hay valores en disputa, los riesgos son altos o las decisiones son urgentes (Funtowicz y Ravetzs, 1993). De este modo, ante decisiones relativas a la economía ecológica (como el calentamiento global) donde existe un cierto nivel de incertidumbre, la mejor manera de rebajarlo es el trabajo en una comunidad extendida de iguales, compuesta por los afectados por un tema en concreto, que estén preparados para entrar en diálogo (Funtowicz y Ravetzs, 1994).

En la evaluación de la sustentabilidad en agroecosistemas el diagnóstico del impacto ambiental debería ser considerado en dos niveles. En el primer nivel o escala, correspondiente a la unidad básica, se encuentra la granja o predio agrícola, en la que los agricultores deben buscar optimizar la producción, con la mínima utilización de insumos y emisión de contaminantes (Wei y Julius, 2001). En un nivel superior es posible 
realizar la evaluación regional de la agricultura y ganadería referidas a una entidad geográfica, diferenciada y estructurada en función de los grupos sociales que habitan e interactúan en ella, situación que hace que los límites geográficos de una región agrícola sean demasiado variables, ya que dependerá de los factores políticos, económicos y sociales considerados (Payraudeau y van der Werf, 2005).

Yunlong y Smith (1994) han sugerido, por otra parte, que la sostenibilidad de la agricultura y ganadería debe ser evaluada desde la perspectiva de la solidez de los sistemas ecológicos, la aceptación social y la viabilidad económica. La solidez ecológica se refiere a la conservación y mejora del medio ambiente natural. La viabilidad económica apunta al mantenimiento de los rendimientos y la productividad de los cultivos y del ganado, y la aceptabilidad social hace mención a la autonomía, la igualdad y la mejora de la calidad de vida. A pesar de existir un amplio consenso acerca de las características básicas de la agricultura sostenible, hay menos consenso sobre qué componentes deberían tener más importancia en la evaluación de la sostenibilidad. Es así cómo, dependiendo del origen académico, algunos profesionales destacan los aspectos ecológicos de la agricultura sostenible tales como el mantenimiento de la salud agroecológica, la diversidad biológica, el manejo integrado de nutrientes, y la calidad del paisaje; mientras que los que atribuyen importancia a los aspectos económicos de la sostenibilidad, evalúan parámetros tales como el valor actual neto, la relación costo/ beneficio y la rentabilidad (Rasul y Thapa, 2004).

Ghersa et al. (2002) señala tres maneras de inferir la sostenibilidad a nivel de explotación agropecuaria:

i. Evaluación del nivel de energía extraída (EE) del sistema. Lo que se resume a través de la siguiente fórmula:

$$
\mathrm{EE}=\mathrm{EMP}+\mathrm{EGP}
$$

Donde:

EMP: Energía exportada por la producción animal en cada explotación (Mcal-ha-1).

EGP: Energía exportada por la producción agrícola en cada explotación (Mcal-ha-1).

Considerándose que la sostenibilidad disminuye en la medida que EE aumenta.

ii. Evaluación de nivel de fragmentación. En este aspecto, se considera que se realiza un uso más sostenible de la tierra agrícola en la medida que no haya reducción de hábitat, o cuando, se evite o disminuya la fragmentación provocada por la agricultura y ganadería por medio de la combinación de atributos (como parches, pasillos o mosaicos) que aumentan la conectividad entre parches de hábitats naturales.

iii. Evaluación de la sostenibilidad agrícola y ganadera dentro de un paisaje. Como es bien sabido, las prácticas agropecuarias generan efectos negativos sobre los ecosistemas, (por ejemplo la erosión de los suelos, la contaminación del suelo y del agua o la reducción de la biodiversidad), efectos que se relacionan en forma directa con las entradas al sistema (laboreo, fertilizantes, herbicidas, plaguicidas) y, en general, con la intensificación (presión de pastoreo en ganadería). Esta relación fue utilizada para evaluar la sostenibilidad, dado que mientras más entradas, para lograr un determinado nivel de producción, mayor es el riesgo de influir negativamente en un ecosistema.

\section{INDICADORESE ÍNDICES}

En la evaluación de la sustentabilidad en general y en el caso particular de su aplicación en los agroecosistemas, la herramienta básica de evaluación (desde la perspectiva de la sustentabilidad fuerte) son los indicadores y, por supuesto, su agregación en índices.

Los indicadores e índices son cada vez más reconocidos como una herramienta útil para la formulación de políticas y comunicaciones públicas como medio de transmitir información sobre el comportamiento de los 
países en ámbitos como el medio ambiente, la economía, la sociedad o el desarrollo tecnológico (Singh et al., 2009). Consisten en medidas sencillas, la mayoría cuantitativas, que representan un estado de los derechos económicos, sociales y/o del desarrollo del medio ambiente en una región definida, a menudo en el plano nacional. Cuando los indicadores son agregados de alguna manera (sistema de indicadores), la medida resultante es un índice (Ness et al., 2007).

En la literatura, los criterios de selección de indicadores de sustentabilidad (o de desarrollo sostenible) adecuados que constituyen la base para cualquier índice de desarrollo sostenible se han discutido ampliamente (OECD, 1994; Atkinson et al., 1997; Hodge y Hardi, 1997 y Böhringer y Jochem, 2007).

Böhringer y Jochem (2007) resumen que la selección de indicadores debe basarse en los siguientes criterios:

a) rigurosa conexión con las definiciones de sustentabilidad

b) que permitan una percepción holística

c) fiabilidad y disponibilidad de cuantificar datos a lo largo de horizontes temporales

d) adecuación al proceso que se desea valorar

e) posibilidad de tener (sub) objetivos políticos derivados

f) adecuada normalización, agregación, y ponderación de las variables

Tanto en indicadores como en índices de sustentabilidad se distinguen dos categorías:

Convencionales del desempeño de una sociedad. Que analizan el comportamiento global del sistema económico, entre los que encontramos aquellos relacionados con la tasa de empleo y desempleo, el consumo, la producción de bienes y servicios y el nivel de precios al consumidor. Algunos ejemplos son el Producto Interno Bruto (PIB) y el Índice de desarrollo Humano (IDH).

De sustentabilidad propiamente tal. En esta categoría, la sustentabilidad débil em- plea indicadores o índices monetarios de sustentabilidad, entre los que se puede mencionar el PIB Verde y el Índice de Bienestar Económico Sustentable; en tanto que la sustentabilidad fuerte, utiliza indicadores o índices biofísicos de sustentabilidad entre los que se encuentran la Huella Ecológica y el Îndice de Planeta Vivo.

\section{INDICADORES}

Los indicadores ambientales son signos de cambio que reflejan, en la dinámica del desarrollo, condiciones específicas de las transformaciones sistémicas del componente ambiental y su incidencia en la alteración del bienestar social del hombre y demás seres vivos. Para un mayor significado en la evaluación de la sustentabilidad ambiental, se establecen indicadores con base en parámetros que permiten una visión holística de la naturaleza, y en relación con la supervivencia del hombre. Los indicadores, son signos de evaluación ambiental que se generan del análisis de los procesos que utilizan recursos ambientales para producir resultados. Se obtienen mediante una serie de parámetros específicos que, considerados en su conjunto y no de manera aislada, determinan la eficiencia, la economía, la equidad y el impacto ambiental (López y Mantilla, 2006).

La OECD (2002a) define indicador como "una variable que describe una característica del estado de un sistema, generalmente a través de datos observados o estimados". Algunos indicadores de acuerdo con Mayer (2008) pueden informar acerca de la posición del sistema particular en relación con límites u objetivos de la sustentabilidad (indicadores de distancia al objetivo) o como indican Hodge et al. (1999) proporcionan señales para medir el progreso hacia objetivos que contribuyen conjuntamente al bienestar humano y al bienestar de los ecosistemas. Es importante señalar que un indicador no dice nada acerca de la sustentabilidad, si no hay un valor de referencia, como por ejemplo los límites y situaciones 
entre los que varían sus valores (Lancker y Nijkamp, 2000).

Es importante señalar la diferencia entre indicadores ambientales e indicadores de sustentabilidad; mientras que los primeros señalan el estado y variación del medioambiente, los indicadores de sustentabilidad indican además, el estado y la variación del sistema humano en relación con el sistema natural (Jiménez-Herrero, 2001). Por ejemplo, en una empresa dedicada a la producción de leche bovina se produjo en un año $1 \mathrm{~kg} / \mathrm{ha} /$ año de gases con efecto invernadero (indicador ambiental); un indicador de sustentabilidad sería el propósito de la empresa de reducir esta cantidad en un $20 \%$ en el próximo año. Es decir, un indicador medioambiental se convierte en uno de sustentabilidad con la adición de los factores de tiempo, límites y objetivos (Meadows, 1998).

La característica principal de los indicadores es su capacidad para resumir, condensar y centrar la enorme complejidad del entorno dinámico a una cantidad manejable de información significativa (Godfrey y Todd, 2001). Por tanto, los indicadores de sustentabilidad permiten la visualización de fenómenos, poniendo de relieve las tendencias, lo que permite simplificar, cuantificar, analizar y comunicar de un modo más simple información compleja (Singh et al., 2009). Además proporcionan información clave acerca de un sistema físico, social o económico, permitiendo el análisis de las tendencias y de las relaciones causa-y-efecto $\mathrm{y}$, por tanto, dando un paso más allá de los datos primarios (Veleva y Ellenbecker, 2001).

En resumen, según Singh et al. (2009), los indicadores de sustentabilidad pueden ser utilizados para:

a) anticipar y evaluar las condiciones y tendencias

b) proporcionar información de alerta temprana para prevenir daños económicos, sociales y medioambientales

c) formular estrategias y comunicar ideas

d) apoyar la adopción de decisiones.

Tabla I. Valores cualitativos de indicadores de sustentabilidad en ecosistemas naturales, en agroecosistemas modernos y en agroecosistemas sustentables. (Qualitative values of sustainability indicators in natural ecosystems, agroecosystems modern and sustainable agroecosystems).

\begin{tabular}{lccc}
\hline Indicador & $\begin{array}{c}\text { Ecosistema } \\
\text { natural }\end{array}$ & $\begin{array}{c}\text { Agroecosistema } \\
\text { moderno }\end{array}$ & $\begin{array}{c}\text { Agroecosistema } \\
\text { sustentable }\end{array}$ \\
\hline $\begin{array}{l}\text { Productividad } \\
\text { Diversidad de especies }\end{array}$ & Media & Alta & Media (Posiblemente alta) \\
Diversidad funcional & Alta & Baja & Media \\
Estabilidad de output & Alta & Baja & Media-alta \\
Acumulación de biomasa & Media & Media-baja & Alta \\
Reciclaje de nutrientes & Alta & Baja & Media-alta \\
Relaciones tróficas & Cerrado & Abierto & Semicerrado \\
Regulación de la población natural & Complejas & Simple & Intermedio \\
Resiliencia & Alta & Baja & Media-alta \\
Dependencia de input externos & Alta & Baja & Media \\
Reemplazo humano de procesos ecológicos & Baja & Blta & Media \\
Sustentabilidad & Alta & Alta & Media-baja \\
\hline
\end{tabular}

Fuente: Pretty (2008) 
EVALUACIÓNDE LA SUSTENTABILIDADEN AGROECOSISTEMAS

Tabla II. Indicadores de uso de recursos e impacto ambiental en ganaderías danesas. (Indicators of resource use and environmental impact in Danish herds).

\begin{tabular}{|c|c|c|}
\hline Tópico & Razón, localización del impacto potencial & Indicador \\
\hline Nitrógeno (N) & $\begin{array}{l}\text { Contaminación de aguas subterráneas, } \\
\text { eutrofización de aguas superficiales y } \\
\text { marinas }\end{array}$ & $\begin{array}{l}\text { Superávit, } \mathrm{kg} \mathrm{N} \mathrm{ha}^{-1} \\
\text { Eficiencia del } \mathrm{N} \text { ( } \mathrm{kg} \text { de } \mathrm{N} \text { en producto } \\
\text { vendido por } \mathrm{kg} \text { de } \mathrm{N} \text { ingresado) }\end{array}$ \\
\hline Fósforo (P) & $\begin{array}{l}\text { Recurso limitado, riesgo de } \\
\text { contaminación a largo plazo }\end{array}$ & $\begin{array}{l}\text { Superávit, kg P ha-1 } \\
\text { Eficiencia del } \mathrm{P} \text { ( } \mathrm{kg} \text { de } \mathrm{P} \text { en producto } \\
\text { vendido por } \mathrm{kg} \text { de } \mathrm{P} \text { ingresado) }\end{array}$ \\
\hline Energía & $\begin{array}{l}\text { Recurso limitado, contaminación, } \\
\mathrm{CO}_{2} \text {, etc. }\end{array}$ & $\begin{array}{l}\text { MJ ha-1 } \\
\text { MJ kg-1 leche o carne } \\
\text { MJ kg-1 grano }\end{array}$ \\
\hline Pesticidas & $\begin{array}{l}\text { Contaminación de aguas subterráneas y } \\
\text { superficiales (especialmente no corrientes) } \\
\text { Flora y fauna salvaje }\end{array}$ & Frecuencia de tratamiento \\
\hline Suelo & $\begin{array}{l}\text { Paisaje estético } \\
\text { Contaminación } \\
\text { Estructura del suelo }\end{array}$ & $\begin{array}{l}\text { \% de área no cultivada (biotopos) } \\
\text { Excedente de } \mathrm{Cu}, \mathrm{kg} \mathrm{ha}^{-1} \text { (granjas } \\
\text { porcinas) }\end{array}$ \\
\hline
\end{tabular}

Fuente: Halberg (1999).

Como la medición del progreso de sociedades complejas y con múltiples dimensiones de calidad de vida a través de un único enfoque, resulta absurdo, Henderson (1994) señala la necesidad del desarrollo de indicadores interdisciplinarios que no son sólo requeridos por economistas, sino por estadísticos de muchas disciplinas (por ejemplo, en la asistencia sanitaria en la Organización Mundial de la Salud(OMS), en materia de educación, alfabetización, etc. en la UNESCO, en estadísticas del impacto ambiental de los consumidores en la Unión Internacional de Consumidores o en materia de medio ambiente en el Programa de las Naciones Unidas para el Medio Ambiente: PNUMA).

En la tabla I es posible apreciar indicadores cualitativos empleados en ecosistemas naturales, agroecosistemas modernos y agroecosistemas sustentables (Pretty, 2008).
Indicadores cuantitativos han sido utilizados por ejemplo por Halberg (1999) para evaluar el uso de recursos y el impacto ambiental en ganaderías danesas, considerando aspectos tales como fertilización, uso de pesticidas y características del suelo (tabla II).

En lo referente a los indicadores relacionados con los aspectos mencionados en la tabla II, se señala la existencia de algunos indicadores más complejos, a modo de ejemplo, en el caso de los pesticidas, se destaca el EPRIP (Environmental Potential Risk Indicator for Pesticide: Indicador de riesgo ambiental potencial de pesticidas), que considera en su determinación la relación entre la toxicidad y la exposición a las concentraciones ambientales previstas, estimando a escala local (de campo y alrededores) y a corto plazo parámetros toxicológicos como DL50 y NOEL (Trevisan et al., 2009). 
Hanegraaf et al. (1998), por otra parte, avanzaron un paso más al desarrollar una metodología para evaluar la sustentabilidad ecológica y socio-económica de cultivos de biomasa, definiendo 12 indicadores ecológicos y 3 socio-económicos para tal efecto, entre los que se destacan los relacionados con el balance de energía, la emisión de gases con efecto invernadero, la incorporación de minerales al suelo y agua, diseminación de pesticidas, la producción y utilización de desechos, costo de la energía producida, costo de disminuir la emisión de $\mathrm{CO}_{2}$ y creación de empleo por hectárea.

ÍNDICES

Un índice es una agregación cuantitativa de indicadores que puede proporcionar una visión simplificada, coherente y multidimensional de un sistema, (Mayer, 2008), lo que resulta muy útil para centrar la atención y, a menudo simplificar el problema (Atkinson et al., 1997).

Para la elaboración de un índice, en primer lugar se debe considerar el número y la naturaleza de los componentes que lo formarán, determinando estos en función de la teoría, el análisis empírico, el pragmatismo, el instinto, o alguna combinación de los mismos. En la figura 3 es posible apreciar esquemáticamente el proceso de construcción de un índice.

Los índices se basan en indicadores que no necesariamente tienen una unidad común de medida, situación que dificulta la forma de ponderación. Otro problema de los índices es su subjetividad, originada en los supuestos de la estimación del error de los datos, en los mecanismos de inclusión o exclusión de indicadores, en la transformación y recorte de indicadores, en el plan de normalización, en la elección de la imputación del algoritmo de cálculo, la elección de las ponderaciones y la elección del sistema de agregación (Singh et al., 2009). Los diversos métodos para el cálculo de índices son detallados por OECD (2002b, c).

Si bien los métodos pueden parecer di- ferentes, en realidad, todos ellos poseen algunas características comunes (Munda, 2005):

a) Todos los índices se basan en el supuesto del establecimiento de una escala de medición para los fines de agregación (dinero, energía, espacio, etc.). Esto crea la necesidad de tomar decisiones muy fuertes en los supuestos de los coeficientes de conversión que se utilizarán y hasta qué punto las actuaciones económicas pueden causar la destrucción del medio ambiente o la exclusión social.

b) El objetivo de la política a menudo no es claro. Las comparaciones entre países o ciudades diferentes son los objetivos de la política de gestión de sostenibilidad de un determinado país o ciudad. En este último caso, un ejercicio de evaluación comparativa se convierte en esencial. Los índices agregados son algo confusos, si se desea obtener sugerencias de política.

c) La mayor parte de los métodos pertenecen a la familia más general de los indicadores compuestos y como consecuencia, las hipótesis utilizadas para su construcción son comunes a todos ellos.

Un completo resumen de los índices utilizados en las áreas de la innovación, conocimiento y tecnología, de desarrollo, comercio y economía, ecosistema, industria, sustentabilidad de ciudades, políticas medioambientales nacionales y regionales, entre otras puede encontrarse en Singh et al., (2009).

A continuación se describen algunos de los índices comúnmente utilizados a nivel mundial.

\section{Huella ecológica(HE)}

La huella ecológica definida en 1996 por Rees y Wackernagel (1996), es un indicador de carácter integrador del impacto que ejerce una cierta comunidad humana, país, región o ciudad sobre su entorno. Corresponde al área de terreno necesaria para producir los recursos consumidos y para asimilar los residuos generados por una población de- 
terminada con un modo de vida específico, donde quiera que se encuentre esa área. Este indicador se sustenta sobre la base cuantitativa de tierra y el agua necesarias para mantener un nivel de vida en lo infinito, por lo que asume ciertas mejoras en la eficiencia. El cálculo de la HE se basa en los datos de estadísticas nacionales de consumo, de esta manera consiste en la normalización de cualquier consumo convertido en el uso de la tierra (Böhringer y Jochen, 2007).

Según World Wildlife Fund for Nature se calcula que la huella ecológica mundial ha superado la capacidad ecológica del mundo en alrededor de un 25\% (WWF, 2006). En promedio la huella ecológica per capita de los países de ingresos altos durante el año 2003 fue de 6,4 ha/persona, lo que supera dramáticamente la disponibilidad per capita de los recursos productivos de la tierra, que en ese año era de sólo 1,8 ha/persona (WWF, 2006). En la figura 2 es posible apreciar la evolución de la huella ecológica durante las últimas 4 décadas.

\section{Índice de Desarrollo Humano (IDH)}

Desde 1990, el Índice de Desarrollo Humano (IDH), por supuesto aplicable al análisis y comparación de sistemas agrarios o ganaderos, o entre sectores productivos, se informa anualmente como parte del Informe sobre Desarrollo Humano de las Naciones Unidas para el Desarrollo (UNDP, 2005). Este consta de tres sub-índices de igual ponderación que son agregados por una media aritmética:

- Índice de Esperanza de Vida al nacimiento

- Índice de Educación que se divide en tasa de alfabetización de adultos (con dos tercios de peso) y una combinación de la tasa bruta de matrícula de la enseñanza primaria, secundaria y terciaria (con un tercio de peso)

- PIB per cápita (en dólares de los EE.UU.)

Estos índices se formulan sobre la base de valores mínimos y máximos para cada indicador y el desempeño en cada dimensión se expresa como un valor entre 0 y 1 . El

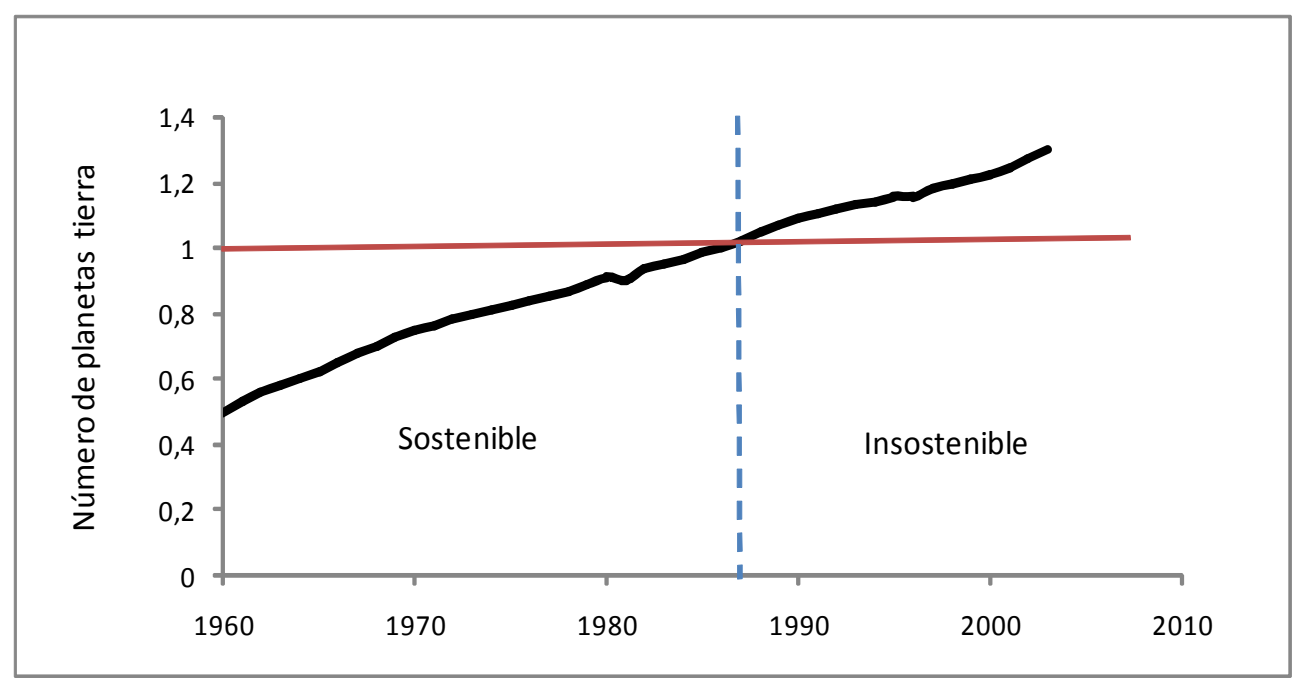

Fuente: Adaptado de World Wide Fund for Nature, (2006).

Figura 2. Huella Ecológica de la humanidad (1961-2003). (Humanity's Ecological Footprint (1961-2003)). 
IDH presenta un fuerte énfasis en la dimensión social del desarrollo sostenible (Singh et al., 2009 y Böhringer y Jachem, 2007).

\section{Índice de planeta vivo (IPV).}

El Índice Planeta Vivo, que fue desarrollado por la WWF en el año 1998, mide las tendencias en la diversidad biológica de la Tierra. Lleva el registro de las poblaciones de 1313 especies de vertebrados, peces, anfibios, reptiles, aves, mamíferos, de todos los lugares del mundo. Se generan índices separados para las especies terrestres, marinas y de agua dulce y luego se promedian las tres tendencias para generar un índice agregado (WWF, 2006). Dentro de cada tendencia se observan todas las especies y se calcula la relación entre pares de poblaciones en años consecutivos (Singh et al., 2009). Aunque los vertebrados representan solamente una fracción de las especies conocidas, se supone que las tendencias en sus poblaciones son típicas de la biodiversidad en general (WWF, 2006).

Al rastrear las especies silvestres, el Índice Planeta Vivo también monitorea la salud del ecosistema. Entre 1970 y 2003, este índice cayó en un 30 por ciento. La tendencia global sugiere que estamos degradando los ecosistemas naturales a un ritmo sin precedentes en la historia de la humanidad (WWF, 2006).

\section{Producto Interno Bruto (PIB)}

Corresponde a un indicador netamente económico, ya que deja de lado todos los efectos ambientales que se producen durante el proceso productivo. Representa la suma del valor añadido de todos los productos de una economía. El PIB a precios de mercado (PIBpm) es destinado al gasto en la demanda de bienes y servicios de consumo final (C) y de bienes de inversión (Formación Bruta de Capital: FBC), esto es, la demanda interna agregada. A esta demanda interna si se le añade el saldo exterior (exportaciones, $\mathrm{E}$, menos importaciones, I) se obtiene el Producto Interno Bruto a precios de merca- do (PIBpm), según la siguiente expresión:

$$
\mathrm{PIBpm}=\mathrm{C}+\mathrm{FBC}+\mathrm{E}-\mathrm{I}
$$

El PIBpm, es producto porque mide la producción final; es interno, por obtenerse en el territorio del país, con independencia de que los propietarios de los factores sean o no residentes; es bruto, porque no contabiliza la depreciación del capital utilizado para generarlo y por último, es a precios de mercado, porque incorpora al valor de la producción los impuestos que la gravan y no deduce de su coste las subvenciones que se le conceden (Fernández, 2006).

\section{PIB Verde}

El PIB Verde fue desarrollado en el ámbito de aplicación de SCAEI (Sistema Integrado de Contabilidad Ambiental y Económica-PNUMA, 2000 y Naciones Unidas, 2003 citado por Singh et al., 2009). Su objetivo es reflejar en los indicadores macroeconómicos de producción (PIB, Producto Nacional Neto, Producto Nacional Bruto) una serie de ajustes, particularmente la depreciación sufrida por los activos medioambientales y los efectos sociales derivados del aumento de la contaminación ambiental, que no son contabilizados en el PIB convencional de los Sistemas de Cuentas Nacionales (Fernández, 2006).

Existen al menos tres formas de obtener el PIB Verde (Hanley, 2000):

- restando las depreciaciones de los recursos naturales causados por su extracción de la Renta Racional Neta.

- restando a la Renta Nacional Neta los gastos necesarios para alcanzar el mismo estado del medio ambiente al final del período.

- restando los gastos de presión sobre el medioambiente y destrucción (calculados por los métodos de la disposición a pagar).

La agregación de los valores obtenidos en cada método se realiza mediante la adición simple de los valores monetarios. 


\section{EVALUACIÓN DE LA SUSTENTABILIDADENAGROECOSISTEMAS}

\section{Índice de Bienestar Económico Sustenta- ble (ISEW)}

Fue propuesto por Daly y Cobb en 1989 (Index of Sustainable Economic Welfare: ISEW). Este índice introduce elementos no monetarizados que aumentan el bienestar general, así como elementos que lo disminuyen como gastos defensivos públicos por degradación ambiental y depreciación del capital natural (Fernández, 2006).

El primer paso del enfoque metodológico de ISEW es la ponderación de los gastos privados dada una población con un índice de desigualdad de ingresos. Posteriormente esta cifra es modificada mediante la suma y resta del valor monetario de un determinado conjunto de factores que se considera afectan el bienestar general de la población tanto en una forma positiva como negativa, así por ejemplo el gasto realizado en actividades recreativas (que aumentan el bienestar), son consideradas positivas, en tanto que gastos originados por la contaminación de agua, aire o suelo (que lo disminuyen), son consideradas negativas. Un aumento de los niveles de ISEW a lo largo del tiempo implica el aumento del bienestar y un progreso positivo hacia la sustentabilidad, por esto, las medidas que promuevan este aumento deben ser preferidas (Gasparatos et al., 2008).

Aunque distintos autores han calculado el bienestar económico sostenible de diversas maneras, los componentes básicos pueden ser generalizados en los siguientes (Dietz y Neumayer, 2007):

$$
\mathrm{BES}=\mathrm{CP}+\mathrm{TR}+\mathrm{GPND}+\mathrm{GDP}-\mathrm{DGF}-
$$
CDA -DRN + AC

\section{Donde:}

BES: Bienestar económico sustentable

$\mathrm{CP}$ : Consumo personal ponderado por la desigualdad de ingresos, que puede considerarse como el dinero que los individuos están dispuestos a pagar con el fin de incrementar su bienestar con la compra de bienes y servicios (Jackson y Stymne, 1996)
TR: Trabajo doméstico, se relaciona con el gasto que se produce al realizar actividades cotidianas fuera de casa, como por ejemplo comer. GPND: Gasto público no-defensivo, a diferencia del gasto defensivo, que es aquel que se hace para mantener o no perder bienestar, el gasto no defensivo es aquel que lleva a un incremento del bienestar (Castañeda, 1999).

GDP: Gastos de defensa privado. Gastos personales que no contribuyen a incrementar el bienestar, por ejemplo gastos de transporte, gastos por accidentes, contaminación, etc. (Cobb y Cobb, 1994).

DGF: Diferencia entre los gastos en consumo duraderos y flujos de servicios de consumo duraderos.

CAD: Los costes de la degradación del medioambiente. Consiste en los costos de contaminación de agua y aire, y de los costos derivados del cambio climático y destrucción de la capa de ozono, que reflejan el daño ambiental a largo plazo (Jackson y Stymne, 1996).

DRN: La depreciación de los recursos naturales. Este término, ligado estrechamente a la sustentabilidad, considera el efecto que el consumo del capital natural hoy ejerce sobre la disponibilidad de recursos en las generaciones futuras (Daly y Cobb, 1989).

AC: Ajustes de capital. Se incluye este término para considerar los bienes de consumo durables y de este modo ver el crecimiento real del capital.

\section{ENFOQUE AGROPECUARIO DE LOS ÍNDICES GLOBALES}

Los indicadores anteriormente mencionados al ser expresados en términos globales no se relacionan directamente con los sistemas de producción agropecuaria. No obstante, la interiorización en ellos hace posible apreciar su utilidad y relación con estos sistemas. Es así como, en el caso de la Huella Ecológica para su cálculo han sido utilizadas, entre otras, las hectáreas necesarias para el consumo de alimentos de origen vegetal y animal. Si por ejemplo nos centramos en el componente alimentos de origen animal y sabemos que como promedio un argentino consume $57 \mathrm{~kg}$ de carne de vacuno y la productividad promedio de la 
actividad es de $200 \mathrm{~kg} / \mathrm{ha} / \mathrm{año}$, se tiene que para sostener este consumo se necesitan 0,285 ha por habitante. Como se puede deducir del ejemplo anterior, los valores de la Huella Ecológica, dependen directamente del tipo de alimento consumido así como de la productividad y eficiencia en las actividades agropecuarias involucradas en su generación.

Por otro lado, si se considera el Índice de Desarrollo Humano, a nivel global, existe una cierta relación entre el porcentaje de población rural y los valores de este índice, por ejemplo comparando países extremos como Bélgica $(0,953)$ y Etiopía $(0,414)$, con porcentaje de población rural de 3 y $84 \%$ respectivamente, es posible apreciar una abismante desigualdad. Si bien es cierto, se está ignorando un sinnúmero de otras variables que distinguen a estos países, la diferencia de este índice en áreas más símiles (como por ejemplo regiones dentro de un país), podría ser utilizada como un indicador social de la sustentabilidad de agroecosistemas.

Situaciones similares ocurren para IPV, PIB, PIB Verde e ISEW, dado que al cambiar la escala de evaluación, es propicia su aplicación en agroecosistemas como índices de sustentabilidad económica, social y medioambiental según corresponda. En el caso del IPV, es preciso mencionar, que el tipo de sistema de producción (tradicional, intensiva, ecológica o sustentable) tiene un impacto directo sobre este índice, dado su efecto en la diversidad de especies y respeto a la conservación de nichos ecológicos naturales.

\section{EVALUACIÓNDE SUSTENTABILIDAD ENAGROECOSISTEMAS}

La evaluación de la sustentabilidad en ecosistemas agrícolas y pecuarios debe ser enfocada desde sus tres dimensiones (económica, social y ambiental). En la dimensión económica deben evaluarse la rentabilidad, los márgenes de producción, costos me- dios, gastos en alimentación e insumos sanitarios y los costos en mano de obra. Dentro de la dimensión social, destaca la generación de empleo, las condiciones de trabajo y, con ello, la disminución de la tasa de inmigración en sectores rurales. Finalmente, la evaluación de la sustentabilidad en la dimensión ecológica o ambiental se orienta a determinar el impacto del manejo de cultivos y la producción pecuaria en los recursos agua, suelo y aire. Se debe señalar, que dentro de la dimensión económica de encuentran en forma implícita los aspectos técnicos de la producción, por lo tanto esta dimensión será enfocada como una dimensión técnico-económica.

\section{INDICADORESDESUSTENTABILIDADTÉCNICO- ECONÓMICAENAGROECOSISTEMAS.}

Como señalan Viglizzo y Roberto (1989) los principales objetivos del administrador agropecuario son el aumento de los retornos económicos y al mismo tiempo, la reducción de los riesgos asociados con la granja. De este modo, los indicadores de sustentabilidad técnico-económica deben asociarse con la obtención de dichos objetivos.

Considerando la sustentabilidad técnica, inicialmente pueden tenerse en cuenta los indicadores relacionados con el manejo o uso de recursos dentro de la explotación, como por ejemplo concentrado consumido $\mathrm{x}$ animal ${ }^{-1}$, superficie $\mathrm{x} \mathrm{cabra}{ }^{-1}$, mano de obra x 100 cabras $^{-1}$ (Ruiz et al., 2008), uso del agua (van Calker et al., 2004), entre muchos otros.

Por el lado de los outputs obtenidos, es preciso mencionar los indicadores relacionados con la productividad utilizados por diversos autores (Viglizzo y Roberto, 1989; Ghersa et al., 2002; Ruíz et al., 2008), que pueden expresarse en variadas unidades, abarcando aspectos de cantidad y calidad, en función del tipo de producción y el objetivo de la investigación. A modo de ejemplo, se pueden mencionar indicadores relacionados con la cantidad de producto tales como crías vendidas x cabra-1 (Nahed et al., 
2006), litros de leche $x$ vaca $^{-1}$, ganancia de peso $\mathrm{x}$ cerdo $^{-1}$, kgMS $\mathrm{x} \mathrm{ha}^{-1}$, Mca $\mathrm{x} \mathrm{ha}^{-1}$ (Ghersa et al., 2002), kg de concentrado $\mathrm{x}$ litro de leche ${ }^{-1}$ (Nahed et al., 2006), y relacionados con calidad como gramos de proteína $\mathrm{x} \mathrm{kg}$ de leche ${ }^{-1}$ (Nahed et al., 2006), gramos de grasa y proteína $\mathrm{x} \mathrm{kg} \mathrm{de} \mathrm{leche-1} \mathrm{(Del} \mathrm{Prado}$ y Scholefield, 2008), rendimiento de cultivos (Belcher et al., 2004), etc. Okey (1996) asocia el concepto de productividad con el de eficiencia, señalando que esta última describe el output obtenido por unidad de input, definición que puede ser transformada a beneficio o ingreso neto por unidad de recurso cuando es enfocada desde la dimensión económica(Conway, 1987). En este sentido Van Calker et al. (2005) seleccionan a la rentabilidad como único atributo para la medición de la sostenibilidad económica, valorando ésta como el retorno anual de la mano de obra familiar, del capital propio o de la gestión, en tanto que Van Calker et al. (2008) utilizan el ingreso neto de la granja como indicador de la sustentabilidad económica de lecherías bovinas holandesas. Cosio (1999), de manera similar, en sistemas ganaderos trashumantes, toma como indicadores los ingresos totales por unidad animal expresados en dólares. Por su parte, Hanegraaf et al. (1998) al evaluar la sustentabilidad económica de cultivos energéticos incorporan el precio de costo de la energía producida, que es valorada en relación a la energía gastada en los procesos de cultivo, cosecha, transporte, secado y almacenaje, y los costos de procesamiento y conversión.

Para estimar indicadores relacionados con el nivel de producción además de la obtención directa de datos, se han utilizado ecuaciones, que por ejemplo relacionan carga animal con producción de carne por hectárea (Viglizzo, 1982) o estrategia nutricional con producción de leche (Herrero et al., 1999), entre otros.

Fernández (2005) recurre al análisis en conjunto de tres indicadores de sustentabilidad técnica: nivel de productividad, nivel de estabilidad y nivel de sostenibilidad; estimando dichos niveles por medio de la utilización de funciones Cobb-Douglas.

Luego, a través de la recopilación de ecuaciones que relacionan las distintas variables de entradas de los agroecosistemas, algunos autores han generado modelos de simulación para predecir variables utilizadas como indicadores de sustentabilidad, ejemplos de esto se pueden apreciar en Vayssières et al. (2009), Del Prado y Scholefield (2008) y Díaz-Solis et al. (2003), donde a través de modelos de simulación, en el primer caso, se determinaron tres indicadores de sustentabilidad técnica: producción de ensilaje, producción de forraje y la producción de leche; en el segundo la cantidad y calidad de la leche y en el tercero la producción primaria neta de forraje.

\section{INDICADORESDESUSTENTABILIDADECOLÓGI- CA-AMBIENTALENAGROECOSISTEMAS.}

La evaluación de sustentabilidad desde esta perspectiva debe centrarse en indicadores relacionados con calidad del suelo, calidad del agua, calidad del aire balance de nutrientes, composición botánica y biomasa total. Así, en la determinación de la calidad del suelo Shukla et al. (2006) por medio de un análisis factorial se seleccionaron nueve factores que pueden ser utilizados para evaluar la sustentabilidad del uso y las prácticas de manejo del suelo en agroecosistemas. En la misma línea, Niño de Zepeda (1994) propone un indicador cuantitativo de impacto ambiental, que se basa en dos variables: la pérdida de suelo por erosión hídrica y el ingreso de materia orgánica al sistema. En el ámbito del balance de nutrientes, son generalmente evaluados $\mathrm{N}$, P y K; para determinar el balance de estos minerales Lértora et al. (1998) utilizan un modelo matemático simple, estimando la diferencia entre las principales pérdidas y ganancias, a través de la cuantificación del contenido de nutrientes de cada producto (en $\mathrm{g} \mathrm{x} \mathrm{kg}^{-1}$ de producto). Van Calker et al. (2004) amplían las variables relacionadas 
con la sustentabilidad, incluyendo siete indicadores de sustentabilidad ambiental en un modelo de programación lineal, estos indicadores junto con los atributos a los cuales representan se detallan en la tabla III.

Castoldi y Benchini (2010) utilizan indicadores de rotaciones de cultivos, cubierta de suelo, carbono orgánico en el suelo, balance de nitrógeno y fósforo en la superficie del suelo, entrada, salida y ganancia de energía y toxicidad de pesticidas dentro de la dimensión ecológica de la evaluación, añadiendo luego la dimensión económica, en la construcción de un índice de sustentabilidad de sistemas de cultivo, para comparar explotaciones en el norte de Italia. Dichos autores representan esquemáticamente la generación de este índice como se muestra en la figura 3.

Para la determinación de algunos indicadores ecológico-ambientales específicos se han realizado trabajos que han generado ecuaciones de regresión. Entre ellos se puede mencionar los trabajos realizados por de Boer et al. (2002) y Monteny et al. (2002) para predecir la emisión de amonio desde establos lecheros.

Al igual que en el caso de indicadores de sustentabilidad técnica-económica, para la determinación de los indicadores de sustentabilidad ecológico-ambiental, también se han utilizado modelos de simulación. Así Alary et al. (2008), a través de un modelo de simulación, además de indicado- res de sustentabilidad técnico-económicos y sociales, determinan indicadores ecológico-ambientales, como la eficiencia de utilización de nitrógeno y el exceso de nitrógeno almacenado en la explotación. Olesen et al. (2006), utilizan un modelo para simular el flujo y la oferta de nitrógeno y las emisiones de gases con efecto invernadero en un conjunto de granjas lecheras Europeas, comparando granjas convencionales y orgánicas, estimando que tipo de producción presenta mejores indicadores en relación a la dimensión ecológico-ambiental de la sustentabilidad. Belcher et al. (2004) utilizan un modelo de evaluación de la sustentabilidad en agroecosistemas, considerando indicadores ecológico-ambientales relacionados con la calidad del suelo, las emisiones de $\mathrm{CO}_{2} \mathrm{y}$ la rotación de cultivos, para analizar los resultados desde dos perspectivas, comparando la sostenibilidad de cada agroecosistema en relación con los demás, o examinando si cada sistema es administrado en forma sostenible en relación con un punto de referencia compuesto de condiciones ambientales y económicas iniciales.

En resumen, es posible identificar un sinnúmero de indicadores dentro de esta dimensión que es, indudablemente, la que presenta mayor complejidad en su proceso de evaluación. Sin embargo, existen ciertos criterios básicos que deben cumplir los indicadores ecológico-medioambientales:

- Evaluar el estado y la capacidad de

Tabla III. Atributos e indicadores para sustentabilidad ecológica utilizados en granjas lecheras bovinas holandesas. (Attributes and indicators for ecological sustainability with respect to Dutch dairy farming).

\begin{tabular}{ll}
\hline Atributos & Indicadores \\
\hline Eutrofización & Eutrofización potencial por hectárea \\
Contaminación de aguas subterráneas & Concentración de $\mathrm{NO}_{3}$ - en aguas subterráneas \\
Deshidratación del suelo & Uso del agua $\left(\mathrm{m}^{3} / \mathrm{ha}\right)$ \\
Acidificación & Acidificación potencial por hectárea \\
Calentamiento global & Calentamiento global potencial por hectárea \\
Ecotoxicidad & Ecotoxicidad potencial acuática por hectárea \\
& Ecotoxicidad potencial terrestre por hectárea \\
\hline
\end{tabular}

Archivos de zootecnia vol. 59 (R), p. 86. 


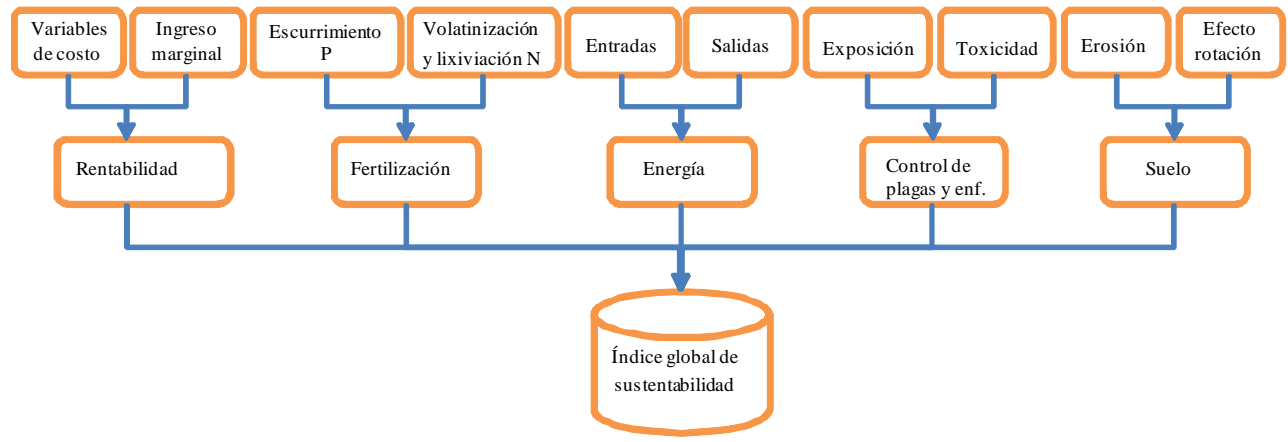

Fuente: Adaptado de Castoldi y Benchini (2009).

Figura 3. Diagrama de flujo de un procedimiento de agregación de indicadores en un índice. (Flow chart of a procedure for aggregation of indicators in an index).

mantener o recuperan la capacidad productiva del suelo y la calidad de agua y aire

- evaluar el estado y la capacidad de conservación de los recursos naturales

- evaluar el grado de diversificación de sistema productivos

- incorporar las relaciones vinculantes, sinérgicas o transversales que se producen entre los componentes del capital natural.

\section{INDICADORES DE SUSTENTABILIDAD SOCIAL ENAGROECOSISTEMAS.}

Como señalan Hutchins y Sutherland (2008), el pilar asociado con la dimensión social de la sustentabilidad no ha sido bien definido. La discusión de este elemento ha recibido poca atención en la literatura, donde se hace hincapié más bien en las cuestiones legislativas, en la salud y la seguridad humana y no en consecuencias culturales y éticas de las decisiones. Dentro de las consecuencias éticas, la equidad es un concepto que ha sido señalado como una de las propiedades socioeconómicas puras que debe cumplir un agroecosistema socialmente sustentable (Okey, 1996) y que se refiere a la uniformidad tanto en la distribución espacial como temporal de productos como al acceso a inputs agrícolas y ganaderos dentro del agroecosistema (Zhen y Routray, 2003).
Zhen y Routray (2003) señalan la igualdad de acceso a recursos como tierras de cultivo, agua de riego, y servicios de apoyo tales como los servicios de extensión y capacitación, comercialización, y crédito de los agricultores y ganaderos como factores que garantizan la sustentabilidad social dentro de un agroecosistema. Algunos de los indicadores sociales relacionados con estos factores, pueden ser observados en el set de indicadores para agricultura sustentable diseñado por MAFF (2000) (tabla IV), en los que se incluyen además otros como la edad de los administradores agroganaderos, el ingreso promedio de los trabajadores, el empleo agrario y el conocimiento del código de buenas prácticas agrarias (Conway, 1987). Fernandes y Woodhouse (2008) añaden a la lista otros cuatro indicadores para la evaluación de esta dimensión de la sustentabilidad en granjas familiares en el sur de Brasil, estos correspondieron al promedio de escolaridad del hogar, el acceso a crédito, las redes técnicas y el índice de electricidad.

Van Calker et al. (2006) al identificar los indicadores de sustentabilidad social dentro de una granja lechera agregan un componente adicional al dividirlos en internos y externos, considerando dentro de los primeros a los relacionados con las condiciones de trabajo y denominando externos a 
los relacionados con la seguridad alimentaria, bienestar y salud animal y calidad del paisaje.

\section{Perspectivadeusodelosindicadoresen LAEVALUACIÓNDE AGROECOSISTEMAS.}

En los apartados anteriores se ha mencionado una lista de indicadores de sustentabilidad empleados en la evaluación de agroecosistemas particulares, sin embargo, el sinnúmero de estructuras productivas y criterios empleados hace que dicha lista se incremente día a día, en la medida que se realiza la evaluación de nuevos sistemas de producción. Esta situación, a pesar, de la posibilidad de generación de indicadores pertinentes, impide la comparación del grado de sustentabilidad entre sistemas pro- ductivos evaluados en forma atemporal o entre sistemas disímiles. Así mismo, el grado de complejidad con que cada indicador es obtenido, por ejemplo a través de medidas de campo, modelos matemáticos y modelos de simulación, también presenta inconvenientes en la medida que se busque la comparación entre sistemas evaluados por distintas metodologías.

Muchos de los indicadores mencionados carecen de la capacidad de predecir el estado y la variación del sistema humano con el sistema natural, debiendo ser mirados en conjunto con otros indicadores para la obtención de dichas propiedades, complicando la comprensión de los resultados.

Las falencias en la medición realizada por algunos indicadores, en busca de la

Tabla IV. Lista de indicadores de sustentabilidad agrícola de MAFF (2000). (List of indicators of agricultural sustainability of MAFF (2000)).

\begin{tabular}{|c|c|c|c|}
\hline $\mathrm{N}^{\mathrm{O}}$ & Indicadores & $\mathrm{N}^{\mathrm{O}}$ & Indicadores \\
\hline 1 & Activos y pasivos agrícolas & 20 & Manejo del estiércol \\
\hline 2 & Edad de los agricultores & 21 & Emisiones agrícolas de amonio \\
\hline 3 & Porcentaje de explotaciones arrendadas & 22 & Emisiones agrícolas de metano y óxido \\
\hline 4 & Ayuda estimada de la UE a los agricultores & & nitroso \\
\hline 5 & $\begin{array}{l}\text { Pago a los agricultores para fines agroam- } \\
\text { bientales }\end{array}$ & $\begin{array}{l}23 \\
24\end{array}$ & $\begin{array}{l}\text { Consumo directo de energía por las granjas } \\
\text { Tendencia en la entrada de energía }\end{array}$ \\
\hline 6 & Ingresos totales por granja & & indirecta a la agricultura \\
\hline 7 & $\begin{array}{l}\text { Ingreso promedio de los trabajadores } \\
\text { agrícolas }\end{array}$ & $\begin{array}{l}25 \\
26\end{array}$ & $\begin{array}{l}\text { Uso de agua para riego } \\
\text { Contenido de materia orgánica en la }\end{array}$ \\
\hline 8 & Productividad agrícola & & cubierta superficial de los suelos agrícolas \\
\hline 9 & Empleo agrícola & 27 & Acumulación de metales pesados en la \\
\hline 10 & $\begin{array}{l}\text { Adopción de sistemas de gestión de } \\
\text { explotaciones }\end{array}$ & 28 & $\begin{array}{l}\text { cubierta superficial de suelos agrícolas } \\
\text { Área de tierras agrícolas }\end{array}$ \\
\hline 11 & Zona convertida a la agricultura ecológica & 29 & Cambio en el uso de la tierra de la agricultu \\
\hline 12 & $\begin{array}{l}\text { Conocimiento del código de buenas } \\
\text { prácticas agrícolas }\end{array}$ & 30 & $\begin{array}{l}\text { ra a usos urbanos } \\
\text { Plantación de cultivos no alimentarios }\end{array}$ \\
\hline 13 & Pesticidas en ríos & 31 & Área de tierras agrarias en virtud del \\
\hline 14 & Pesticidas en aguas subterráneas & & compromiso con la conservación del medio \\
\hline 15 & $\begin{array}{l}\text { Cantidad de ingredientes activos de } \\
\text { plaguicidas utilizados }\end{array}$ & 32 & $\begin{array}{l}\text { ambiente } \\
\text { Características de las tierras de cultivo }\end{array}$ \\
\hline 16 & Área tratada con pesticidas & 33 & Área en los márgenes de los cultivos de \\
\hline 17 & Residuos de pesticidas en alimentos. & & cereales bajo conservación medioambiental. \\
\hline 18 & Pérdidas de nitratos y P en la agricultura & & Área de pastizales semi-naturales \\
\hline 19 & Niveles de P en la capa superior del suelo & 33 & Poblaciones de aves agrícolas claves \\
\hline
\end{tabular}

Fuente: MAFF (2000).

Archivos de zootecnia vol. 59 (R), p. 88. 
simplificación de las mismas, no deja de ser un inconveniente, a modo de ejemplo se puede mencionar la medición que Lértora et al., (1998) utilizan para el balance de nutrientes, estimando la diferencia entre las principales pérdidas y ganancias de un sistema, a través de la cuantificación del contenido de nutrientes de cada producto, dejando de lado pérdidas tan importantes de nutrientes como las de lixiviación, erosión o volatilización.

En el ámbito de la sustentabilidad social, la medición a escala predial se hace aún más confusa, dados los distintos regímenes de propiedad, formas jurídicas y tipos de explotación (familiar o no familiar) que determinan los objetivos de las empresas agropecuarias y con ello la estructuración de su mano de obra.

Por otro lado, la desigualdad en número, así como en el grado de profundidad de los indicadores utilizados dentro de cada dimensión, genera un desequilibrio en la evaluación produciendo un cierto sesgo en los resultados. Del mismo modo, se precisa la existencia de una cierta complementariedad entre los indicadores de distintas dimensiones. A modo de ejemplo, si consideramos la productividad (kg de carne/ha) de un predio ganadero extensivo dentro de la dimensión técnico económica, debemos considerar dentro de la dimensión ecológica- ambiental una medida que se relacione con la disponibilidad de pasto y demás problemas asociados con el sobrepastoreo como por ejemplo la composición botánica.

\section{MARCOSDE SUSTENTABILIDADEN AGROECOSISTEMAS.}

Un marco de sustentabilidad no es un índice, ya que no implica ninguna agregación cuantitativa de los datos, sino más bien agregaciones cualitativas que proporcionan maneras de presentar un gran número de indicadores (Olalla-Tárraga, 2006). Por ejemplo, el marco de Presión-Estado-Respuesta (PSR) es un grupo de indicadores que describen las presiones o estrés de los sistemas o recursos (por ejemplo, la intensidad de la tala o la intensidad del pastoreo), la situación del sistema de recursos (por ejemplo, la superficie forestal, o ganadera), y los esfuerzos de los habitantes o los gobiernos para mejorar la situación (por ejemplo, las leyes contra la tala ilegal o restricción de la carga ganadera en algunos sistemas productivos ) (Mayer, 2008). Otro marco bastante complejo es el CRITINC, que posee indicadores divididos en tres niveles y agrupados por la dimensión y el tipo de función representada en los ecosistemas (Ekins et al., 2003). Otros marcos utilizan un enfoque visual para identificar los patrones en los indicadores. Por ejemplo, el Dashboard de la Sustentabilidad presenta los indicadores en una matriz similar, a los indicadores de colores, del tablero de control de un avión. Otros marcos gráficos incluyen el AMOEBA y el Barómetro de la Sustentabilidad (Mayer, 2008). Este tipo de marcos entregan de forma rápida una situación general del sistema en estudio, ya que poseen la ventaja con respecto a los índices de que todos los valores de los indicadores son fácilmente observados y no están ocultos detrás de un índice agregado, no existiendo pérdida de información.

La metodología MESMIS (Marco de evaluación de sustentabilidad basado en indicadores) combina las ventajas de algunos de los marcos mencionados anteriormente (Masera et al., 1999). Su estructura operativa se basa en un ciclo de seis pasos, siendo los tres primeros destinados a la caracterización del sistema, identificación de puntos críticos, y definición de los indicadores pertinentes para cada uno de los siete atributos con los que define sustentabilidad (productividad, estabilidad, resiliencia, confiabilidad, adaptabilidad, equidad y autogestión). Los tres pasos siguientes se encargan de la integración de los indicadores definidos (por medio de técnicas cualitativas, cuantitativas o multicriterio), con el propósito de obtener una 
visión integrada (ya sea un índice o un enfoque visual como el AMOEBA) de la sustentabilidad del o los sistemas evaluados (López-Ridaura etal., 2002, 2005). Ejemplos de la aplicación de esta metodología a sistemas agropecuarios pueden ser encontrados en Bowen y Valenzuela (2009), Gaspar et al.,(2009), Speelman et al., (2007) y Nahed et al, (2006), entre otros.

\section{CONCLUSIONES}

En agroecosistemas, las metodologías de evaluación generan indicadores netamente dependientes del sistema en estudio, lo que condiciona una especificidad y la dificultad de una implementación general. Metodologías como la MESMIS presentan un alto grado de flexibilidad permitiendo su adaptación a diversos agroecosistemas por medio de la definición de puntos críticos, criterios de diagnóstico e indicadores pertinentes. Esta metodología cumple la mayor

\section{BIBLIOGRAFÍA}

Alary, V., Gousseff, M. and Nidumolu, U.B. 2008. Comparison of multi-criteria decision models to approach the trade-off between environmental sustainability and economical viability - a case of nitrogen balance in dairy farming systems in Reunion Island. J. Agric. Sci., 146: 389-402.

Atkinson, G., Dubourg, R., Hamilton, K., Munasignhe, M., Pearce, D. and Young, C. 1997. Measuring sustainable development: macroeconomics and the environment. Edward Elgar Publishisng. Cheltenham, UK.

Belcher, K., Boehm, M. and Fulton, M. 2004. Agroecosystem sustainability: a system simulation model approach. Agric. Syst., 79: 225-241.

Bell, M. 1998. An invitattion to environmental sociology. Pine Forge Press. Londres.

Beloff, B. and Beaver, E. 2000. Sustainability indicators and metrics of industrial performance. Paper SPE 60982. Presentado en SPE International Conference on Health, Safety, and Environment in Oil and Gas Exploration and parte de los requerimientos establecidos por Brink (1991) y Beloff y Beaver (2000); destacando su utilidad en la evaluación de sistemas que se sustentan en la utilización de razas autóctonas o locales y que se localizan en zonas desfavorecidas y marginales.

A pesar de los avances en la determinación de sustentabilidad, la necesidad de definir y emplear evaluaciones, tanto para la creación y aplicación de políticas gubernamentales, como para el apoyo a la toma de decisiones a nivel predial sigue siendo una tarea pendiente en gran parte de los sistemas de producción agropecuarios de Latinoamérica.

\section{AGRADECIMIENTOS}

La autora principal agradece a la Agencia Española de Cooperación Internacional por el aporte financiero recibido durante la realización de sus estudios de Postgrado.
Production. Stavanger. Noruega.

Böhringer, C. and Jochem, P. 2007. Measuring the immeasurable. A survey of sustainability indices. Ecol. Econ., 63: 1-8.

Bowen, S. and Valenzuela, A. 2009. Geographical indications, terroir, and socioeconomic and ecological sustainability: The case of tequila. $\mathrm{J}$. Rural Stud., 25: 108-119.

Brink, B. 1991. The AMOEBA approach as a useful tool for establishing sustainable development. In: Kuik, O.,Verbruggen, H. (Eds.). In search of indicators of sustainable development. Kluwer Academic Publishers. The Netherlands.

Castañeda, B. 1999. An Index of Sustainable Economic Welfare (ISEW) for Chile. Ecol. Econ., 28: 231-244.

Castoldi, N. and Bechini, L. 2010. Integrated sustainability assessment of cropping systems with agro-ecological and economic indicators in northern Italy. Eur. J. Agron. 32: 59-72.

Cobb, C.W. and Cobb, J.B. 1994. The green national product. University Press of America. New

Archivos de zootecnia vol. 59 (R), p. 90. 


\section{EVALUACIÓN DE LA SUSTENTABILIDADENAGROECOSISTEMAS}

York. 343 pp.

Conway, G.R. 1987. The properties of agroecosystems. Agric. Syst., 24: 95-117.

Cosio, F. 1999. Determinación y análisis de la estructura técnico-económica para la asignación de derechos de uso de pastizales en la veranada de montaña. Laguna del Maule. Chile. Tesis. Universidad de Córdoba. Córdoba. España. $285 \mathrm{pp}$.

Costanza, R., and Patten, B. 1995. Commentary: defining and predicting sustainability. Ecol. Econ., 15: 193-196.

Daly, H. and Cobb, J. 1989. For the common good. Beacon Press. Boston.

De Boer, I., Smits, M., Mollenhorst, H., van Duinkerken, G. and Monteny, G. 2002. Prediction of ammonia emission from dairy barns using feed characteristics. Part I: Relation between feed characteristics and urinary urea concentration. J. Dairy Sci., 85: 3382-3388.

Del Prado, A. and Scholefield, D. 2008. Use of SIMSDAIRY modelling framework system to compare the scope on the sustainability of a dairy farm of animal and plant genetic-based improvements with management-based changes. J. Agric. Sci., 146: 195-211.

Devuyst, D., Hens, L. and Lannoy, W. 2001. How green is the city? Sustainability assessment and the management of urban environments. Columbia University Press. New York.

Díaz-Solis, H., Kothmannb, M., Hamiltonb, W. and Grantc, W. 2003. A simple ecological sustainability simulator (SESS) for stocking rate management on semiarid grazinglands. Agric. Syst., 76: 655-680.

Dietz, S. and Neumayer, E. 2007. Weak and strong sustainability in the SEEA: concepts and measurement. Ecol. Econ., 61: 617-626.

Ekins, P., Simon, S., Deutsch, L., Folke, C. and De Groot, R. 2003. A framework for the practical application of the concepts of critical natural capital and strong sustainability. Ecol. Econ., 44: 165-185.

Fernandes, L.A.d.O. and Woodhouse, P.J. 2008. Family farm sustainability in southern Brazil: an application of agri-environmental indicators. Ecol. Econ., 66: 243-257.

Fernández, G. 2005. Alternativas de gestión con ganadería bovina en sistemas pastoriles de la Región Semiárida Pampera (Argentina). Siste- ma de cría y engorde de la propia producción. Tesis. Universidad de Córdoba. Córdoba. España. p. 277.

Fernández, F. 2006. Indicadores de sostenibilidad y medio ambiente: métodos y escala. Junta de Andalucía. Consejería de Medio Ambiente. Sevilla. España.

FMAM. 2000. Contribuciones del fondo para el medio ambiente mundial al programa 21. www.gefweb.org. (22-6-09).

Funtowicz, S. and Ravetz, J. 1993. Science for the post-normal age. Futures, 25: 39-755.

Funtowicz, S. and Ravetz, J. 1994. The worth of a songbird: ecological economics as a postnormal science. Ecol. Econ., 10: 197-207.

Gaspar, P., Mesías, F., Escribano, M. and Pulido, F. 2009. Sustainability in spanish extensive farms (dehesas): An economic and management indicator-based evaluation. Rangel. Ecol. Manag., 62: 153-162.

Gasparatos, A., El-Haram, M. and Horner, M. 2008. A critical review of reductionist approaches for assessing the progress towards sustainability Environ. Impact Assess. Rev., 28: 286-311.

Ghersa, C.M., Ferrano, D.O., Omacini, M., Martínez-Ghersa, M.A., Perelman, S., Satorre, E.H. and Soriano, A. 2002. Farm and landscape level variables as indicators of sustainable land-use in the Argentine Inland-Pampa. Agric. Ecosys. Environ., 93: 279-293.

Godfrey, L. and Todd, C. 2001. Defining thresholds for freshwater sustainability indicators within the context of South African water resource management. $2^{\text {nd }}$ WARFA/Waternet Symposium: Integrated Water Resource Management: Theory, Practice, Cases. Cape Town. South Africa. Disponible en http://ww.waternetonline. ihe.nl/aboutWN/pdf/godfrey.pdf. (6-7-09).

Halberg, N. 1999. Indicators of resource use and environmental impact for use in a decision aid for Danish livestock farmers. Agric., Ecosys. Environ., 76: 17-30.

Hanegraaf, M.C., Biewinga, E.E. and Van der Bijl, G. 1998. Assessing the ecological and economic sustainability of energy crops. Biomass Bioenerg., 15: 345-355.

Hanley, N. 2000. Macroeconomic measures of sustainability. J. Econ. Surv., 14 : 1-30.

Henderson, H. 1994. Paths to sustainable development: the role of social indicators. 


\section{TORO, GARCÍA, GÓMEZ-CASTRO, PEREA, ACERO Y RODRÍGUEZ-ESTÉVEZ}

Futures, 26: 125-137.

Herrero, M., Fawcetta, R. and Dent, J. 1999. Bioeconomic evaluation of dairy farm management scenarios using integrated simulation and multiple-criteria models. Agric. Syst., 62: 169188.

Hodge, A., Hardi, P. and Bell, D. 1999. Seeing change through the lens of sustainability. Paper for the Workshop: Beyond Delusion: Science and Policy Dialogue on Designing Effective Indicators of Sustainable Development. International Institute for Sustainable Development. Costa Rica.

Hodge, R. and Hardi, P. 1997. The need for guidelines: the rationale underlying the bellagio principles for assessment. In: Hardi, P., Zdan, T. (Eds.). Assessing Sustainable Development: Principles in Practice. International Institute for Sustainable Development. Winnipeg. pp. 7-20.

Hutchins, M.J. and Sutherland, J.W. 2008. An exploration of measures of social sustainability and their application to supply chain decisions. J. Clean. Prod., 16: 1688-1698.

Jackson, T. and Stymne, S. 1996. Sustainable economic welfare in Sweden : A pilot index 1950-1992. Stockholm Environmental Institute. Stockholm. Sweden.

Jiménez-Herrero, L. 2001. Desarrollo sostenible y economía ecológica. Integración medio ambiente, desarrollo y economía-ecología. Ediciones Síntesis. Madrid.

Jiménez-Herrero, L. 1989. Medio ambiente y desarrollo sostenible. IEPALA. Barcelona.

Kates, R., Clark, W., Corell, R., Hall, M., Jaeger, C., Lowe, I., McCarthy, J., Schellnhuber, H., Bolin, B., Dickson, N, Faucheux, S., Gallopin, G., Grubler, A., Huntley, B., Jager, J., Jodha, N., Kasperson, R., Mabogunje, A., Matson, P. and Mooney, H. 2001. Sustainability science. Science, 292: 641-642.

Lancker, E. and Nijkamp, P. 2000. A policy scenario analysis of sustainable agricultural development options: a case study for Nepal. Impact Assessment \& Project Appraisal, 18: 111-124.

Lértora, F., Viglizzo, E., Pordomingo, A. and Roberto, Z. 1998. Sistema soporte de decisiones (SSD) para el agro: II. Evaluación de usos de la tierra. Rev. Arg. Prod. Anim., 18: 233.

López, J. y Mantilla, E. 2006. Los indicadores y la medición de a sostenibilidad. En: Mantilla, E.
(Ed.). Medición de la sostenibilidad ambiental. Universidad Cooperativa de Colombia. Colombia. Disponible en http://site.ebrary.com/lib/ bibliocordoba/Doc?id=10154590\&ppg=121 (10-6-09).

López-Ridaura, S., Masera, O. and Astier, M. 2002. Evaluating the sustainability of complex socio-environmental systems. the MESMIS framework. Ecol. Indicators, 2: 135-148.

López-Ridaura, S., Keulen, H., Ittersum, M. and Leffelaar, P. 2005. Multiscale methodological framework to derive criteria and indicators for sustainability evaluation of peasant natural resource management systems. Environ. Dev. Sustain., 7: 51-69.

Luffiego, M, y Rabadán, J. 2000. La Evolución del concepto de sostenibilidad y su introducción en la enseñanza. Enseñanza de las Ciencias, 18: 473-486.

MAFF. 2000. Towards Sustainable Agriculture, A pilot set of indicator. Ministry of Agriculture Fisheries and Food. London.

Masera, O., Asterier, M. and López-Ridaura, S. 1999. Sustentabilidad y manejo de recursos naturales. El marco de la evaluación MESMIS. Grupo Interdisciplinario de Tecnología Rural Apropiada. Ediciones Mundi Prensa. México. $109 \mathrm{pp}$.

Mayer, A. 2008. Strengths and weaknesses of common sustainability indices for multidimensional systems. Environ. Inter., 34: 277-291.

Meadows, D. 1998. Indicators and information systems for sustainable development. A Report to the Balaton Group. The Sustainability Institute. Hartland.

Monteny, G., Smits, M., van Duinkerken, G., Mollenhorst, H. and de Boer, I. 2002. Prediction of ammonia emission from dairy barns using feed characteristics. Part II: Relation between urinary urea concentration and ammonia emission. J. Dairy Sci., 85: 3389-3394.

Munda, G. 2005. Measuring sustainability: A multicriterion framework. Environ. Dev. Sustain., 7: 117-134.

Nahed, J., Castel, J.M., Mena, Y. and Caravaca, F. 2006. Appraisal of the sustainability of dairy goat systems in Southern Spain according to their degree of intensification. Livest. Sci., 101: 10-23.

Archivos de zootecnia vol. 59 (R), p. 92. 


\section{EVALUACIÓN DE LA SUSTENTABILIDADENAGROECOSISTEMAS}

Ness, B., Urbel-Piirsalu, E., Anderberg, S. and Olsson, L. 2007. Survey: Categorizing tools for sustainability assessment. Ecol. Econ., 60: 498508.

Niño de Zepeda, A. 1994. Análisis del conflicto productividad vs. sustentabilidad ambiental: Un enfoque de programación multicriterio. Investigación Agraria: Economía, 9 : 143-155.

OECD (Organization for Economic Cooperation and Development). 1994. Environmental Indicators. OECD. Paris.

OECD (Organization for Economic Cooperation and Development). 2002a. Aggregated environmental indices: review of aggregation methodologies in use. Paris, France: Organization for Economic Co-operation and Development Report No. ENV/EPOC/SE (2001)2/FINAL. 2002. Disponible en http:// www.olis.oecd.org/olis/2001doc.nsf/LinkTo/ env-epoc-se(2001)2-final. (4-5-10).

OECD (Organization for Economic Cooperation and Development). 2002b. OECD Guidelines for Multinational Enterprises. Annual Report 2002.

OECD (Organization for Economic Cooperation and Development). 2002c. An update of the OECD Composite leading Indicators. Short-term economic Statistics division, Statistics Directorate/OECD. Disponible en http:// www.oecd.org/dataoecd/6/2/2410332.pdf. (45-10).

Okey, B. 1996. Systems approaches and properties, and agroecosystem health. J. Environ. Manage., 48: 187-199.

Olalla-Tárraga, M. 2006. A conceptual framework to assess sustainability in urban ecological systems. Int. J. Sust. Dev. World Ecol., 13: 1-15.

Olesen, J., Schelde, K., Weiske, A., Weisbjerg, M., Asman, W. and Djurhuus, J. 2006. Modelling greenhouse gas emissions from European conventional and organic dairy farms. Agric., Ecosyst. Environ., 112: 207-220.

Payraudeau, S. and van der Werf, H.M.G. 2005. Environmental impact assessment for a farming region: a review of methods. Agric., Ecosyst. Environ., 107: 1-19.

Phillis, Y. and Andriantiatsaholiniaina, L. 2001. Sustainability: an ill-defined concept and its assessment using fuzzy logic. Ecol. Econ., 37: 435-456.

Popp, J., Hoag, D. and Hyatt, E. 2001. Sustainability indices with multiple objectives. Ecol. Indicators, 1: 37-47.

Pretty, J. 2008. Agricultural sustainability: concepts, principles and evidence. Phil. Trans. R. Soc. Lond. B. Biol. Sci., 363: 447-465.

Rapport, D., Böhm, G., Buckingham, D., Cairns, J., Costanza, R., Karr, J., de Kruijf, H., Levins, R., McMichael, A., Nielsen, N. and Whitford, W. 1999. Ecosystem health: The concept, the ISEH, and the important tasks ahead. pp. 82-90.

Rasul, G. and Thapa, G. 2004. Sustainability of ecological and conventional agricultural systems in Bangladesh: an assessment based on environmental, economic and social perspectives. Agric. Sys., 79: 327-351.

Rees, W. and Wackernagel, M. 1996. Urban ecological footprints: why cities cannot be sustainable and why they are a key to sustainability. Environ. Impact Assess. Rev., 16: 223-248.

Rigby, D. and Cáceres, D. 2001. Organic farming and the sustainability of agricultural systems. Agric. Sys., 68: 21-40.

Ruiz, F., Castel, J., Mena, Y., Camúñez, J. and González-Redondo, P. 2008. Application of the technico-economic analysis for characterizing, making diagnoses and improving pastoral dairy goat systems in Andalusia (Spain). Small Rum. Res., 77: 208-220.

Shukla, M.K., Lal, R. and Ebinger, M. 2006. Determining soil quality indicators by factor analysis. Soil Till. Res., 87: 194-204.

Singh, R.K., Murty, H.R., Gupta, S.K. and Dikshit, A.K. 2009. An overview of sustainability assessment methodologies. Ecol. Indicat., 9: 189-212.

Smith, F. 1996. Biological diversity, ecosystem stability and economic development. Ecol. Econ., 16: 191-203.

Speelman, E., Lopez-Ridaura, S., Colomer, N.A., Astier, M. and Masera, O. 2007. Ten years of sustainability evaluation using the MESMIS framework: Lessons learned from its application in 28 Latin American case studies. Int. J. Sust. Dev. World Ecol., 14: 345-361.

Trevisan, M., Di Guardo, A. and Balderacchi, M. 2009. An environmental indicator to drive sustainable pest management practices. Environ. Model. Softw., 24: 994-1002.

UNDP (United Nations Development Programme). 
TORO, GARCÍA, GÓMEZ-CASTRO, PEREA, ACERO Y RODRÍGUEZ-ESTÉVEZ

2005. Human development report. Oxford University Press. Oxford.

Van Calker, K.J., Berentsen, P.B.M., de Boer, I.M.J., Giesen, G.W.J. and Huirne, R.B.M. 2004. An LP-model to analyse economic and ecological sustainability on Dutch dairy farms: model presentation and application for experimental farm "de Marke". Agric. Syst., 82: 139-160.

Van Calker, K.J., Berentsen, P.B.M., Giesen, G.W.J., Huirne, R.B.M. 2005. Identifying and ranking attributes that determine sustainability in Dutch dairy farming. Agr. Hum Val., 22: 53-63.

Van Calker, K.J., Berentsen, P.B.M., Giesen, G.W.J. and Huirne, R.B.M. 2008. Maximising sustainability of Dutch dairy farming systems for different stakeholders: A modelling approach. Ecol. Econ., 65: 407-419.

Van Calker, K.J., Berentsen, P.B.M., Romero, C., Giesen, G.W.J. and Huirne, R.B.M. 2006. Development and application of a multi-attribute sustainability function for Dutch dairy farming systems. Ecol. Econ., 57: 640-658.

Vayssières, J., Guerrin, F., Paillat, J.-M. and Lecomte, P. 2009. GAMEDE: A global activity model for evaluating the sustainability of dairy enterprises Part I - Whole-farm dynamic model. Agric. Syst., 101: 128-138.

Veleva, V. and Ellenbecker, M. 2001. Indicators of sustainable production: framework and methodology. J. Clean. Prod., 9: 519-549.

Viglizzo, E. 1982. Los potenciales de produccion de carne en la región pampera semiárida. Prime- ras Jornadas Técnicas sobre Producción Animal en la Región Pampera Semiárida. Facultad de Agronomía. Universidad Nacional de la Pampa. Santa Rosa. La Pampa. Argentina. pp. 233269.

Viglizzo, E.F. and Roberto, Z.E. 1989. Diversification, productivity and stability of agroecosystems in the Semi-Arid Pampas of Argentina. Agric. Syst., 31: 279-290.

Vilches, A., y Gil, D. 2003. Construyamos un futuro sostenible. Diálogos de supervivencia. Cambridge University Presss. Madrid. Capítulo 6.

Wei, X. and Julius, M. 2001. A review of concepts and criteria for assessing agroecosystem health including a preliminary case study of southern Ontario. Agric. Ecosys. Environ., 83: 215-233.

Wei, Y., Davidson, B., Chen, D. and White, R. 2009. Balancing the economic, social and environmental dimensions of agro-ecosystems: An integrated modeling approach. Agric. Ecosys. Environ., 131: 263-273.

WWF (World Wildlife Fund for Nature). 2006. Informe Planeta Vivo 2006. Ed. Hails, C. Gland Suiza.

Yunlong, C. and Smit, B. 1994. Sustainability in agriculture: A general review. Agric. Ecosys. Environ., 49: 299-307.

Zhen, L. and Routray, J.K. 2003. Operational indicators for measuring agricultural sustainability in developing countries. Environ. Manage., 32: 34-46.

Archivos de zootecnia vol. 59 (R), p. 94. 